\section{Pathophysiology of Haemostasis and Thrombosis}

Pathophysiol Haemost Thromb 2005;34:109-120

DOI: $\underline{10.1159 / 000089931}$

\title{
A Model for the Formation and Lysis of Blood Clots
}

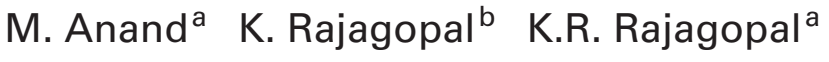 \\ ${ }^{a}$ Department of Mechanical Engineering, Texas A\&M University, College Station, Tex., and \\ ${ }^{\mathrm{b}}$ Department of Surgery, Duke University Medical Center, Durham, N.C., USA
}

\begin{abstract}
Key Words
Hemostasis - Virchow's triad - Platelet activation • Extrinsic pathway $\cdot$ Fibrinolysis $\cdot$ Shear stress
\end{abstract}

\begin{abstract}
Both biochemical and mechanical factors have to be taken into account if a meaningful model for the formation, growth, and lysis of clots in flowing blood is to be developed. Most models that are currently in use neglect one or the other of these factors. We have previously reported a model [J Theoret Med 2003;5:183-218] that we believe is a step in this direction, incorporating many of the crucial biochemical and rheological factors that play a role in the formation, growth, and lysis of clots. While this model takes into account the extrinsic pathway of coagulation, it largely ignores the intrinsic pathway. Here, we discuss some of the general issues with respect to mathematical modeling of thrombus formation and lysis, as well as specific aspects of the model that we have developed.
\end{abstract}

Copyright (C) 2005 S. Karger AG, Basel

\section{Introduction}

A variety of competing biochemical and rheological mechanisms that aid or inhibit clot formation help maintain blood in a state of delicate balance. Coagulation of blood is promoted by the following principal stimuli, characterized nearly 150 years ago by Virchow [2] as: (1) local flow stasis/stagnation, (2) blood vessel injury/endothelial dysfunction, and (3) hypercoagulability, an augmented intrinsic tendency for blood to clot. Clearly, the first cause, local stasis or stagnation, is a consequence of hemodynamic conditions, while the latter two are primarily biochemically driven. However, these factors are interrelated. Clots form when a certain threshold dictated by hemodynamic and biochemical factors is reached, including the flow characteristics (stresses in the fluid), concentrations of various ions, particularly $\mathrm{Ca}^{2+}$, availability of membrane binding sites, and local concentrations of platelets and coagulation factors. Under subthreshold conditions, blood flows in a normal manner, and when the threshold is reached, the balance is shifted towards promotion of coagulation, and in net, a cascade of reactions are set into motion, resulting in the initiation of coagulation. At the end of the hemostatic process, the normal subthreshold conditions are restored. However, pathological conditions may result in either impairment of clotting, thereby leading to bleeding disorders, or hypercoagulability, leading to thrombotic/thromboembolic disorders. These pathological conditions have their origins in both hemodynamic and biochemical factors associated with flowing blood.

Pro- and antithrombotic factors are simultaneously active. These balancing mechanisms serve to maintain blood in the fluid, much less viscous than clot, phase under nomal conditions. Hemodynamic factors play a critical role in modulating biochemical reactions and have to

\section{KARGER}

Fax +4161306 1234

E-Mail karger@karger.ch

www.karger.com
C 2005 S. Karger AG, Basel

$1424-8832 / 05 / 0343-0109 \$ 22.00 / 0$

Accessible online at:

www.karger.com/pht
K.R. Rajagopal

Department of Mechanical Engineering

Texas A\&M University

College Station, TX 77843-3123 (USA)

Tel. +1 979862 4552, Fax +1 979845 3081, E-Mail krajagopal@mengr.tamu.edu 
be taken into consideration in any attempt at explaining the formation, growth and lysis of clots ([3-5] and several of the references mentioned therein.)

The hemostatic system contains both cellular and noncellular components. We now discuss the cellular components. The endothelium plays an essential role in regulating clot formation. First, it forms a physical barrier between blood and the proteins embedded in the procoagulant subendothelial layers. Second, the endothelium serves an important anticoagulant function by virtue of surface expression and release of several molecules including thrombomodulin, prostaglandin $\mathrm{I}_{2}$, and nitric oxide donors.

Platelets are the principal cellular constituent of clots, and critically regulate thrombus formation and lysis. Platelet activation, which is essential for thrombus formation, is notably influenced by well-described biochemical factors as well as by hemodynamic factors. Once platelets are activated, in part by being exposed to shear stresses over a certain critical value for a prolonged time interval, they express and release factors that lead to adhesion to the subendothelial layer as well as platelet-platelet adhesion (aggregation).

The noncellular, plasma phase components comprise the 'coagulation cascade' and related regulatory proteins. Classically, two pathways constituting the system have been described, the extrinsic and the intrinsic. In this paper we shall be primarily concerned with the extrinsic pathway to coagulation, as evidence suggests that it may be the predominant pathway in vivo.

Both the extrinsic and the intrinsic pathway lead to the formation of active thrombin, and the cleavage of fibrinogen that leads to the formation of fibrin monomers that polymerize to form fibrin strands. These fibrin strands form a matrix that binds the platelet aggregates, RBCs, and WBCs, which entrap the plasma and constitute a clot. The fibrin strands themselves, while providing structural integrity to the clot, actually constitute less than $1 \%$ by volume of the clot. Negative regulatory networks also regulate the thrombus formation, including both anticoagulant (inhibitors of coagulation) and destructive (fibrinolytic) mechanisms. However, the numerous biochemical reactions that take place leading to the formation and lysis of clots, and the exact influence of hemodynamic factors in these reactions, are incompletely understood.

A typical example of studies related to the biochemistry of clot formation that are not much concerned with hemodynamic aspects is a recent paper by Kuharsky and Fogelson [6], a detailed study of the roles of surface binding sites and platelet deposition in the context of throm- bus formation. Over fifty reaction-diffusion equations are provided, and they are careful to observe that even these may be insufficient to describe the specific processes in which they are interested. On the other hand, practically all of the papers concerned with hemodynamic factors and hemostasis pay no attention to the myriad of biochemical reactions that take place in flowing blood [7]. We have developed a model [1] that melds together some of the biochemical and rheological aspects; however, our study is incomplete, as it essentially ignores the intrinsic pathway. While the model seems to capture many of the salient features of the phenomenon of clot formation and lysis, as it is an initial skeletal model, it neglects the intrinsic pathway and other specific factors/processes.

Pathologies of clot formation are discussed in the following section by way of motivating the need for this study. After a brief description of the composition of blood, we discuss the changes that occur during, and influences on, platelet activation, the extrinsic and the intrinsic pathway of coagulation, and fibrinolysis in later sections. We then describe constitutive relations to describe the response of normal blood and that of a clot, a condition to capture platelet activation (and aggregation), as also reaction-convection-diffusion equations that govern the biochemical reactions governing coagulation and fibrinolysis, as well as the application of the model to study the formation, growth, and lysis of clots in flowing blood. A brief discussion of the limitations of the model, and extensions, is found in the final section.

\section{Pathologies of Clot Formation}

Pathologies of clot formation, in large part due to abnormalities of coagulation, are responsible for substantial morbidity and mortality. These pathologies can be classified under two broad categories: (1) disorders of pathological thrombus formation and maintenance and (2) disorders leading to impaired thrombus formation, which we now proceed to discuss briefly.

\section{Disorders of Increased Thrombus Formation}

The interested reader can find a more detailed discussion of the disorders of increased thrombus formation, their treatment and references, wherein these matters are discussed, in the literature [1]. Here, we present a brief discussion of these disorders.

Atrial dysrhythmias are the primary cause for intraatrial thrombus formation. These dysrhythmias, such as atrial flutter or fibrillation, are characterized by ineffi- 
cient or absent atrial contraction. Diastolic blood flow into the ventricles is lower and slower than during ventricular systolic ejection under normal circumstances, and this in conjunction with impaired atrial contraction leads to local flow stagnation and consequent thrombus formation. This thrombus formation can result in systemic embolization, a major cause of morbidity and mortality.

Intraventricular cavitary thrombus is less common than intra-atrial thrombus formation by virtue of hemodynamic considerations. However, severe systolic ventricular dysfunction can be associated with thrombus formation. Also, akinetic or dyskinetic (as found in ventricular aneurysms) segments of ventricular myocardium are associated with intracavitary thrombus formation. Thrombi also form on non-endothelialized artificial cardiac valves, though infrequently they can form on native valves.

Acute coronary syndromes and other types of acute arterial insufficiency are two other important pathologies that are typically consequences of intravascular thrombus formation. In the majority of acute coronary syndromes, thrombus forms over unstable or frankly ruptured plaques. However, acute coronary syndromes may arise as a result of a stable plaque's growth causing a critical stenosis, or even due to coronary vasospasm or an acute increase in myocardial oxygen demand that is not met by an increase in oxygen supply. Acute arterial insufficiency of other vascular beds may also be due to thrombus formation over unstable plaque, or due to thrombo- and atheroembolism.

Also of great clinical importance is deep venous thrombosis with or without resultant pulmonary thromboembolism. Flow stasis/stagnation in the deep systemic veins is thought to be the principal hemodynamic inciting factor in the pathogenesis of deep venous thrombosis/pulmonary thromboembolism. Additionally, in accordance with Virchow's triad, underlying hypercoagulability and endothelial injury are also contributing factors.

\section{Bleeding Disorders}

Most bleeding disorders are a consequence of inadequate levels of the components of the hemostatic system or dysfunction of components present at normal levels.

Thrombocytopenia, or a deficiency in blood platelet concentrations, may occur due to impaired platelet synthesis, enhanced platelet sequestration, or platelet destruction. Platelet dysfunction independent of thrombocytopenia also occurs, as is commonly observed in uremia.

A Model for the Formation and Lysis of Blood Clots
Many bleeding disorders are a consequence of reduced level or activity of accessory factors (e.g., von Willebrand factor) or the coagulation factors themselves $[8,9]$. Examples of these are (1) hemophilia A and B, which are X (chromosome)-linked recessively inherited deficiencies in factor VIII and IX, respectively, (2) disease states with global depression of coagulation factor levels due to liver failure and disseminated intravascular coagulation. References to the literature pertaining to such disorders and their treatment can be found in [10].

\section{Blood and the Formation, Growth, and Lysis of a Clot}

\section{Constitution of Blood}

Blood is a complex mixture consisting of gel-like cellular matter in a Newtonian liquid (plasma). The cellular matter normally includes erythrocytes that comprise nearly $98 \%$, leukocytes, and platelets. Plasma is an aqueous solution that contains a variety of proteins that function in the hemostatic system and its regulation: fibrinogen (fI), prothrombin (fII), fV, fVIII, fIX, fX, fXI, fXIII, antithrombin III, tissue factor (TF) pathway inhibitor (TFPI), proteins $\mathrm{C}$ and $\mathrm{S}$, plasminogen (PLS), plasmin (PLA), $\alpha_{1}$-antitrypsin (L1AT), $\alpha_{2}$-antiplasmin (L2AP), and many other constituents. Consistent with its aqueous nature, plasma behaves rheologically as a Newtonian fluid with a viscosity of approximately $1.2 \mathrm{cP}$ [11]. Erythrocytes are enucleated biconcave deformable disks that exhibit viscoelastic behavior, such as those observed in micropipette experiments by Evans and Hochmuth [12]. Leukocytes exhibit viscoelastic behavior [13] . In aggregate, in large blood vessels, blood behaves as a non-Newtonian fluid. However, in capillaries, where blood is effectively separated into cellular and plasma constituents, blood cannot be treated as a homogeneous fluid.

\section{Platelet Activation}

Though platelets form only around 3\% by volume of the cellular material in blood, they play a critical role in the coagulation process. Platelet activation is a consequence of the interactions with collagen and adhesive glycoproteins in the subendothelium, and with soluble thrombin and adenosine diphosphate, when the endothelium is damaged. When activated, the platelet undergoes a series of biochemical and morphological changes, in which the organelles in the platelets are centralized and the glycoproteins that are on the platelet membrane undergo a conformational change so that adhesive pseudo-

Pathophysiol Haemost Thromb 2005;34:109-120 
pods are extended from the platelet surface. These activated platelets (AP) interact with a variety of plasma proteins, such as fIX, fV, von Willebrand factor, fibrinogen, and fibrin, and form aggregates that constitute the clot [14-16]. Platelet activation is also influenced by rheological factors, especially exposure to shear stresses. Several studies have documented the effects of flow conditions on platelet adhesion and deposition, and thrombus formation [17-21].

\section{Extrinsic and Intrinsic Pathways of Coagulation}

Both the extrinsic and intrinsic pathways serve the common purpose of proteolytic cleavage of $\mathrm{fX}$ into the active Xa. It is generally thought that the extrinsic pathway, as it more rapidly initiates coagulation, is the predominant mechanism of $\mathrm{Xa}$ and downstream factor IIa and Ia generation $[22,23]$. However, it is well appreciated that clinical deficiencies in components of the intrinsic pathway, particularly fIX (hemophilia B - Christmas disease) and fVIII (hemophilia A), result in deleterious impairments in coagulation and hemostasis. Factor XI deficiency has also been associated with surgical bleeding [24]. While these observations appear to conflict, some studies have been able to better explain the aforementioned findings. Broadly, these have shown that: (1) components of the intrinsic pathway can be activated by the extrinsic pathway, and (2) once Xa and thrombin have been generated, positive feedback mechanisms can activate the intrinsic pathway. More specifically, using an in vitro reconstitution system without fXII and fXI present, Lawson et al. [25] integrated the findings of previous studies and demonstrated that: (1) the TF/f VIIa complex is both necessary and sufficient for initiation of IIa formation; (2) this correlates with activation of fVIIIa and fVa (added $\mathrm{Va}$ accelerates initiation) to physiological extents, previously shown to be mediated by Xa and IIa, and independent of TF/VIIa; (3) subsequent propagation of IIa formation is dependent upon common pathway zymogen concentrations and concentrations of VIIIa and Va, and (4) increasing concentrations of TF/VIIa can reduce the contribution of fIXa and fVIIIa in overall IIa synthesis, whereas fIXa and fVIIIa contribute substantially to Xa synthesis when TF/VIIa concentrations are low. These data suggest that the extrinsic pathway is essential for the initiation of plasma coagulation as assessed by IIa synthesis, but that the intrinsic pathway can also play a significant role, particularly in situations in which extrinsic pathway activation is not robust; moreover, intrinsic pathway components may be activated by the extrinsic pathway, both directly (via IXa activation) and indirect- ly (via common pathway Xa and IIa feed-forward activation of VIIIa). Whether these experimental findings and resultant models capture coagulation in vivo is unclear, because most studies have been carried out under static (from a fluid mechanics standpoint) conditions.

The initiation and growth of forming clots in spatially inhomogeneous unstirred blood due to both contact activation and activities of the extrinsic pathway has been studied by Ataullakhanov and co-workers [26-30]. The effect of local $\mathrm{Ca}^{2+}$ concentration on the threshold concentration of XIa was examined in [31], and the threshold was experimentally determined by making surface measurements [32]. They did not identify the exact mechanism that inhibited clot growth [33].

Regarding the extrinsic coagulation pathway, exposure of subendothelial TF to flowing blood results in activation of fVII into VIIa, and ultimately leads to thrombin formation. The TF/VIIa complex leads to the formation of fIXa and fXa from their respective plasma zymogens, fIX and fX. The cofactors VIIIa and Va do not have catalytic functions, and are rendered active as cofactors via cleavage by thrombin (IIa). The IXa-VIIIa enzyme complex (tenase) bound to the negatively charged phospholipids catalyzes the formation of Xa from X. This in turn leads to the formation of Xa-Va enzyme complex (prothrombinase) on the membrane of the activated platelet. Prothrombinase catalyzes the conversion of II to IIa. Thrombin in turn acts on plasma fibrinogen to produce fibrin monomers, which are polymerized by fXIII to form the fibrin matrix. These processes are regulated by several inhibitory factors, notably Antithrombin III, tissue factor pathway inhibitor and Proteins $\mathrm{C}$ and S. Excellent discussions of these related issues can be found in the literature [34-37].

Clot formation is directly affected by mechanical factors, such as the shear stresses in flowing blood, and the influx and efflux of substrate, cofactors, and enzymatic catalysis that occurs during blood flow. There is experimental evidence to suggest a dependence of the fibrin formation on the shear rate of flowing blood [38]. Also, fibrin formation occurs in recirculation zones even when the endothelium is intact [39].

\section{Fibrinolysis}

As stated earlier, the negative regulatory networks in the hemostatic system are composed of those factors that inhibit clot formation (anticoagulant; discussed above) and those that actively destroy formed thrombus. Fibrinolysis occurs in parallel with clot formation and growth, and proceeds, in the context of injury, in tandem with 
healing. Thrombin and fibrin activate the endothelial cells in the vicinity of the injured region, resulting in enhanced production of tissue PLS activator (tPA) and urokinase-like PLS activator. tPA and Urokinase-like PLS activator catalyze the conversion of PLS to PLA. PLA degrades the fibrin polymer into smaller units leading to clot lysis. The primary inhibitor of fibrinolysis is $\alpha_{2}$-Antiplasmin. An exhaustive review of the mechanisms of fibrinolysis can be found in the literature [40].

Clot lysis is affected by mechanical factors; in particular, high shear stresses can rupture the clot [41]. This generates clot fragments that may embolize distally.

\section{Mathematical Model for the Formation and Lysis of Clots}

\section{Main Features of the Model}

(1) A model for normal blood as a shear-thinning viscoelastic fluid with a shear-dependent relaxation time, which contains the components involved in, and leading to, the formation, growth, and lysis of the clots.

(2) An activation condition for the platelets that depends on the concentrations of the components as well as the rheological properties of blood.

(3) Development of reaction-convection-diffusion equations for the components that reflect the enzymatic cascade of reactions that leads to clot formation and growth.

(4) Boundary conditions that reflect injury to the blood vessel.

(5) A constitutive model for the clot as a viscoelastic fluid that is shear-thinning, with a relaxation time that is shear-dependent. The clot has a viscosity that is 10 30 times that of normal blood.

(6) A method for tracking the boundary between the growing clot and normal blood.

(7) Reaction-convection-diffusion equations for the components that reflect the fibrinolysis that takes place.

(8) A criterion for the onset of clot lysis based on the concentration of the various components and rheological parameters of the blood and clot.

(9) A viscoelastic fluid model for the lysed clot that is similar to that for normal blood.

\section{Rheological Models for Blood and Clot}

In large vessels blood behaves like a Navier-Stokes (Newtonian) fluid; however, in sufficiently small vessels, the non-Newtonian behavior of blood becomes prominent. This is not surprising, as the diameter of the various components, such as erythrocytes and leukocytes, become of comparable dimension to that of the diameter of the vessel. In fact, in capillaries, the diameter of the vessel can be smaller than the diameter of the erythrocyte (with deformability of the erythrocyte thought to be important to its transit), calling into question the validity of a continuum model for the flowing blood. In view of the properties of the various components of blood, it exhibits a variety of characteristics of a non-Newtonian fluid, namely shear-thinning [11,42], and stress-relaxation [12, $43,44]$. Thurston [45], apart from being the first to note the viscoelastic behavior of blood, also found that the stress-relaxation is more significant at flows that take place at a lower shear rate.

A variety of non-Newtonian fluid models have been used to describe the flow of blood (see [46]). We shall not discuss these models in detail; instead, we shall merely document a more general model that we have previously reported [1], which includes as a special case many of the popular models used for modeling blood. The viscoelastic fluid model of the rate type to describe the response of normal whole blood has sound thermodynamic underpinnings (for details, see [47] concerning the general framework for the development of rate type fluid models and [48] for the specific model for normal whole blood). Here, we shall merely document the constitutive equations for whole blood and clot. The Cauchy stress $\mathbf{T}^{(i)}$ for normal whole blood and clot are given by

$$
\begin{aligned}
& \mathbf{T}^{(i)}=p \mathbf{1}+\mathbf{S}^{(i)}, \\
& \mathbf{S}^{(i)}=\mu^{(i)} \mathbf{B}+\eta^{(i)} \mathbf{D}, \\
& \stackrel{\nabla}{\mathbf{B}}=-2\left(\frac{\mu^{(i)}}{\alpha^{(i)}}\right)^{1+2 n^{(i)}}\left(\operatorname{tr}\left(\mathbf{B}^{(i)}\right)-3 \lambda\right)^{n^{(i)}}\left[\mathbf{B}-\lambda^{(i)} \mathbf{1}\right], \\
& \lambda^{(i)}=\frac{3}{\operatorname{tr}\left[\left(\mathbf{B}^{(i)}\right)^{-1}\right]}, \\
& n^{(i)}=\frac{\gamma^{(i)}-1}{1-2 \gamma^{(i)}}, \quad n^{(i)}>0 .
\end{aligned}
$$

In the above equation $i=1$ stands for normal whole blood and $i=2$ stands for the clot. Also, $\mathbf{B}^{(i)}(i=1,2)$ denotes the Cauchy-Green stretch tensor and $\mathbf{D}^{(i)}$ the symmetric part of the velocity gradient for normal whole blood and clot, respectively. Also, the inverted triangle denotes the Oldroyd derivative [49] defined through

$$
\stackrel{\nabla}{\mathbf{B}}=\dot{\mathbf{B}}-\mathbf{L B}-\mathbf{B L}^{T} \text {, }
$$


where the dot denotes the material time derivative and $\mathbf{L}$ denotes the velocity gradient. Also, $\mu^{(i)}$ denotes the shear modulus, $\eta^{(i)}$ denotes the viscosity, $n^{(i)}$ the shear-thinning index, and $\alpha^{(i)}$ a material constant that describes the evolution of the Cauchy- Green stretch tensor with time. The spherical part of the stress is the reaction due to the assumption that blood is incompressible.

A general thermodynamic framework has been developed to describe the viscoelastic response of fluids [47]. The model documented above has been developed within the above framework. Most classical models due to Maxwell, Burgers, Oldroyd and others can be developed within the context of this framework.

It is important to point out that the models for normal whole blood and clot while similar in that their constitutive expressions have a similar structure, have material functions that describe them that are very different; for example, the viscosity of the clot can be an order of magnitude larger than that for whole blood.

\section{Condition for Platelet Activation}

In mathematical models of platelet activation, activation of the platelets is brought about, in part, by prolonged exposure to suprathreshold values for the shear stresses ( $>5 \mathrm{~Pa}$; see [50,51]) in flowing blood. We assume that the platelets are activated if a parameter, referred to as the activation number, reaches a critical value, denoted by $A_{\text {thr. }}$. We also assume that the lysis of clots occurs when the clots are exposed to shear stresses for a length of time such that the activation number reaches yet another critical number, $A_{\text {damage, }}$, greater in value than the first critical number. That is, the clot is lysed if the activation parameter meets or exceeds $A_{\text {damage }}[1,52]$.

The activation number $A(t)$ is defined through

$$
A(t)=A(0)+\frac{1}{A_{0}} \int_{0}^{t} e^{k\left(\frac{|r z z|}{\tau_{\text {thr }}}-1\right)} H\left(\left|\tau_{r z}\right|-\tau_{\text {thr }}\right) \mathrm{d} t
$$

The criterion for activation of resting platelets (RP) is as follows: If $A\left(t-t_{\text {act }}\right)>A_{\mathrm{thr}}$ and $A(t)<A_{\text {damage }}$ or $A\left(t-t_{\mathrm{act}}\right)=A_{\mathrm{thr}}, \dot{A}\left(t-t_{\mathrm{act}}\right)>0$ and $A(t)<A_{\text {damage }}$, then $[\mathrm{AP}](t)=[\mathrm{AP}]\left(t-t_{\mathrm{act}}\right)+[\mathrm{RP}]\left(t-t_{\mathrm{act}}\right)$.

The criterion for lysis of platelets during activation is as follows: If $A\left(t-t_{\text {act }}\right)>A_{\text {thr }}$ and $A(t)>A_{\text {damage, }}$ then $[\mathrm{AP}](t)=[\mathrm{RP}]\left(t-t_{\mathrm{act}}\right)$.

The values for $A_{0}, t_{\mathrm{act}}, k, A_{\mathrm{thr}}, A_{\text {damage, }}$ etc. can be estimated by fitting our model to the data of Brown et al. [50, 51], Wurzinger et al. [53], and Sorensen et al. [7]. The details concerning the evaluation of these constants can be found in the literature [1, 52].

\section{Model for Coagulation and Fibrinolysis}

Reaction-convection-diffusion equations govern the generation and depletion of each of the $N$ constituents in blood and they take the form

$$
\frac{\partial\left[Y^{(i)}\right]}{\partial t}+\operatorname{div}\left(\left[Y^{Y^{(i)}}\right] \mathbf{v}\right)=\operatorname{diV}\left[D_{Y^{(i)}}(\mathbf{D}) \nabla\left[Y^{(i)}\right]\right]+G_{Y^{(i)}} \quad i=1, \ldots N
$$

where $\left[Y^{(i)}\right]$ denotes the concentration of the $i$-th constituent, $G_{Y^{(i)}}$ denotes its production or depletion due to the enzymatic reactions, v denotes the velocity, and $D_{Y^{(i)}}$ denotes the shear-rate-dependent diffusivity. All these components are assumed to coexist at every point in the flow domain, of course with different concentrations.

In our study, we shall restrict ourselves to the critical constituents that are essential to describe coagulation and fibrinolysis dynamics. We shall be concerned with the extrinsic pathway of coagulation. It is well recognized that the intrinsic pathway at the least augments coagulation due to the extrinsic pathway. We shall assume the following constituents to be essential to describing coagulation and fibrinolysis: resting and activated platelets (RP and AP), fibrinogen and fibrin (I and Ia), prothrombin and thrombin (II and IIa), fV and fVa, fVIII and fVIIIa, fIX and fIXa, fX and fXa, tenase (IXa-VIIIa-AP or ' $\mathrm{Z}$ '), prothrombinase (Xa-Va-AP or ' $\mathrm{W}$ '), fXI and fXIa, antithrombin III, protein $\mathrm{C}$ and activated protein C, TFPI, $\alpha_{1}$-antitrypsin, tPA, PLS and PLA, $\alpha_{2}$-antiplasmin, TF-VIIa complex, and von Willebrand factor. A host of other factors are important and will not be discussed in this study, as even a consideration of all the above factors and the hemodynamic issues leads to a system of highly nonlinear coupled equations. The other factors can be incorporated at a later stage, when some progress has been made in studying the simplified model and assessing its efficacy. We now record the reactionconvection-diffusion equations for the above factors.

$$
\begin{aligned}
& \frac{\mathrm{d}[\mathrm{IX} a]}{\mathrm{d} t}=k_{9}[\mathrm{XI} a]-h_{9}[\mathrm{IX} a]=G_{\mathrm{IX} a}, \\
& \frac{\mathrm{d}[\mathrm{IX}]}{\mathrm{d} t}=-k_{9}[\mathrm{XI} a]=G_{\mathrm{IX}}, \\
& \frac{\mathrm{d}[\mathrm{VIII} a]}{\mathrm{d} t}=k_{9}[\mathrm{II} a]-h_{8}[\mathrm{VIII} a]-k_{a}[\mathrm{APC}]([\mathrm{VIII} a]+[Z]) \\
& =G_{\mathrm{VIII} a} \text {, } \\
& \frac{\mathrm{d}[\mathrm{VIII}]}{\mathrm{d} t}=-k_{8}[\mathrm{II} a]=G_{\mathrm{VIII}},
\end{aligned}
$$




$$
\begin{aligned}
& \frac{\mathrm{d}[\mathrm{Va}]}{\mathrm{d} t}=k_{5}[\mathrm{IIa}]-h_{5}[\mathrm{Va}]-k_{a}[\mathrm{APC}]([\mathrm{Va}]+[W])=G_{\mathrm{Va}}, \\
& \frac{\mathrm{d}[\mathrm{V}]}{\mathrm{d} t}=-k_{5}[\mathrm{IIa}]=G_{\mathrm{V}}, \\
& {[Z]=\frac{k_{8,9}[\mathrm{VIIIa}][\mathrm{IXa}]}{h_{8,9}+k_{a}[\mathrm{APC}]},} \\
& {[W]=\frac{k_{5,10}[\mathrm{Va}][\mathrm{Xa}]}{h_{5,10}+k_{a}[\mathrm{APC}]},} \\
& \frac{\mathrm{d}[\mathrm{Xa}]}{\mathrm{d} t}=k_{10}[\mathrm{Z}]-h_{10}[\mathrm{Xa}]-k_{\mathrm{TFPI}}[\mathrm{TFPI}][\mathrm{Va}]=G_{\mathrm{Xa}}, \\
& \frac{\mathrm{d}[\mathrm{X}]}{\mathrm{d} t}=-k_{10}[\mathrm{Z}]=G_{\mathrm{X}}, \\
& \frac{\mathrm{d}[\mathrm{IIa}]}{\mathrm{d} t}=\frac{k_{2}[W][\mathrm{II}]}{K_{2 m}+[\mathrm{II}]}-h_{2}[\mathrm{IIa}]=G_{\text {IIa }}, \\
& \frac{\mathrm{d}[\mathrm{II}]}{\mathrm{d} t}=\frac{-k_{2}[W][\mathrm{II}]}{K_{2 m}+[\mathrm{II}]}=G_{\mathrm{II}}, \\
& \frac{\mathrm{d}[\mathrm{Ia}]}{\mathrm{d} t}=\frac{k_{1}[\mathrm{IIa}][\mathrm{I}]}{K_{1 m}+[\mathrm{I}]}-\frac{h_{1}[\mathrm{PLA}][\mathrm{Ia}]}{H_{1 m}+[\mathrm{Ia}]}=G_{\text {Ia }}, \\
& \frac{\mathrm{d}[\mathrm{I}]}{\mathrm{d} t}=\frac{-k_{1}[\mathrm{III}][\mathrm{I}]}{K_{1 m}+[\mathrm{I}]}=G_{\mathrm{I}}, \\
& \frac{\mathrm{d}[\mathrm{XIa}]}{\mathrm{d} t}=k_{11}[\mathrm{IIa}]-h_{11}[\mathrm{XIa}]=G_{\mathrm{XIa}}, \\
& \frac{\mathrm{d}[\mathrm{XI}]}{\mathrm{d} t}=-k_{11}[\mathrm{IIa}]=G_{\mathrm{XI}} \\
& \frac{\mathrm{d}[\mathrm{ATIII}]}{\mathrm{d} t}=-h_{9}[\mathrm{IXa}]-h_{10}[\mathrm{Xa}]-h_{2}[\mathrm{IIa}]=G_{\mathrm{ATIII}}, \\
& \frac{\mathrm{d}[\mathrm{TFPI}]}{\mathrm{d} t}=-k_{\mathrm{TFPI}}[\mathrm{TFPI}][\mathrm{Xa}]=G_{\text {TFPI }}, \\
& \frac{\mathrm{d}[\mathrm{APC}]}{\mathrm{d} t}=k_{\mathrm{APC}}[\mathrm{IIa}]-h_{\mathrm{APC}}[\mathrm{APC}]=G_{\mathrm{APC}}, \\
& \frac{\mathrm{d}[\mathrm{PC}]}{\mathrm{d} t}=-k_{\mathrm{APC}}[\mathrm{IIa}]=G_{\mathrm{PC}}, \\
& \frac{\mathrm{d}[\mathrm{L} 1 \mathrm{AT}]}{\mathrm{d} t}=-h_{\mathrm{APC}}[\mathrm{APC}]=G_{\mathrm{LIAT}}, \\
& \frac{\mathrm{d}[\mathrm{tPA}]}{\mathrm{d} t}=0=G_{\mathrm{tPA}} \\
& \frac{\mathrm{d}[\mathrm{PLA}]}{\mathrm{d} t}=k_{\mathrm{PLA}}^{\mathrm{tPA}-\mathrm{Ia}}[\mathrm{tPA}]-h_{\mathrm{PLA}}[\mathrm{PLA}][\mathrm{L} 2 \mathrm{AP}]=G_{\mathrm{PLA}},
\end{aligned}
$$

$$
\begin{aligned}
& \frac{\mathrm{d}[\mathrm{PLS}]}{\mathrm{d} t}=-k_{\mathrm{PLA}}^{\mathrm{tPA}-\mathrm{Ia}}[\mathrm{tPA}]=G_{\mathrm{PLS}}, \\
& \frac{\mathrm{d}[\mathrm{L} 2 \mathrm{AP}]}{\mathrm{dt}}=-h_{\mathrm{PLA}}[\mathrm{PLA}][\mathrm{L} 2 \mathrm{AP}]=G_{\mathrm{L} 2 \mathrm{AP}} .
\end{aligned}
$$

In general, the coefficients ( $k$ 's) in the above equations are not constant but functions of the various concentrations and kinematical quantities. We shall assume them to be constants here.

\section{Application of Model to Study Clot Formation, \\ Growth, and Lysis in Flowing Blood}

The reaction-convection-diffusion equations have to be solved in conjunction with the balance laws for mass, linear and angular momentum, and energy. The constitutive relations 1-2 automatically satisfy the balance of angular momentum as the choice for the Cauchy stress tensor is symmetric.

The balance of mass is given by

$$
\frac{\partial \rho}{\partial t}+\operatorname{div}(\rho \mathbf{v})=0
$$

and the balance of linear momentum is given by

$$
\operatorname{div} \mathbf{T}+\rho b=\rho \frac{\mathrm{d} \mathbf{v}}{\mathrm{d} t},
$$

while the balance of energy takes the form

$$
\rho \frac{\mathrm{d} \varepsilon}{\mathrm{d} t}=\mathbf{T} \mathbf{L}-\operatorname{div} \mathbf{q}+\rho r .
$$

In the above equation $\rho$ denotes the density, $\mathbf{b}$ the specific body force, $\varepsilon$ the specific internal energy, $\mathbf{q}$ the heat flux vector, $\mathbf{L}$ the velocity gradient, and $r$ the radiant heating. Since blood has been assumed to be incompressible, it can undergo only isochoric motions, and hence it has to meet

$\operatorname{div} \mathbf{v}=0$.

Finally, the second law of thermodynamics takes the form

$$
\text { T.L }-\rho \dot{\psi}-\rho \eta \dot{\sigma}-\frac{\text { q.grad } \sigma}{\sigma}=\rho \sigma \xi:=\zeta \geq 0 .
$$

In the above inequality $\psi$ denotes the specific Helmholtz energy, $\sigma$ the absolute temperature, $\eta$ the specific entropy, and $\xi$ is the rate of entropy production. Our choices of the constitutive quantities have to be such that they always satisfy the second law of thermodynamics; the choices we make in the study do so $[48,54]$.

Under normal circumstances, no clots exist, and we assume the constitutive equation for normal blood. A clot is defined to have been formed if the concentration of fi- 
Fig. 1. Spatial evolution of fibrin concentration during the formation, growth, and lysis of a clot in quiescent plasma (with platelets) on a thrombogenic plane.

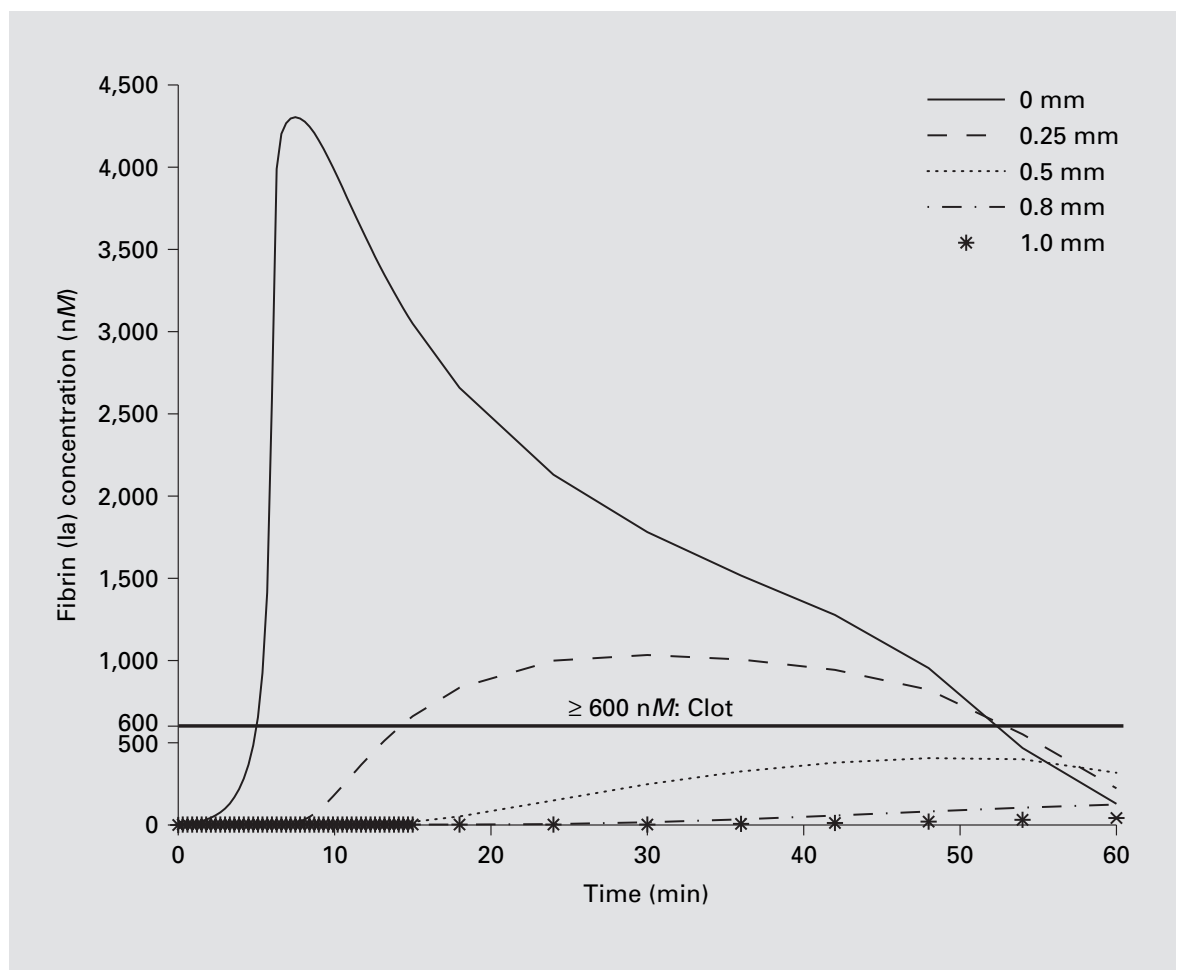

brin exceeds $600 \mathrm{n} M$ at any point in the flow domain. The growth of the clot can then be tracked by determining the extent of the region wherein the fibrin concentration exceeds $600 \mathrm{n} M$. In this region we assume the constitutive relation for the clot. If at a later instant of time the fibrin concentration falls below $600 \mathrm{n} M$ in a region which was originally occupied by the clot, we state that clot lysis has taken place and we revert to using the original model that we used for normal blood. In order to be in a position to switch between the models in the different domains, we need to solve all of the balance and reaction-convectiondiffusion equations simultaneously and track the concentration of fibrin.

There are numerous reaction rate constants and diffusion coefficients that appear in the reaction-convectiondiffusion equations 7-31. Many of these constants have been measured through independent experiments, others have been estimated assuming the solute is undergoing Brownian motion. A discussion of how a reasonable choice can be made for the various constants that appear in the equations 7-31 can be found in the literature (see tables 1-4 in [1]). Choices have to be made for the zero shear viscosity, the infinite shear viscosity, relaxation time, and the other constants that appear in the constitutive equation for blood. Values that are pertinent to these material parameters for human blood are available in the literature [1].

Using these values for the various reaction rate constants, diffusion coefficients, and constitutive parameters, we studied (1) the problem of clot formation, growth, and lysis in quiescent plasma (with platelets) on a thrombogenic plane and (2) the case of time varying fully developed Poiseuille flow of blood in a cylindrical domain with a view towards corroborating the model with an experimental program. The model is able to predict the formation of a clot, track its growth (which leads to a free boundary problem for the boundary of the clot) and its lysis, and the results seem to be physically reasonable. However, at the current time, there are no experimental data available to meaningfully evaluate the efficacy of the model.

The case of clot formation, growth, and lysis in quiescent plasma (with platelets) on a thrombogenic plane involves solving only the reaction-diffusion equations with the appropriate boundary conditions (the boundary conditions, in this case, are the simultaneous generation of TF/VIIa and XIa and also for the generation, as soon as thrombin is produced, of tPA to initiate fibrinolysis). The spatial and temporal dynamics of fibrin (Ia) concentration (and thus of the clot, as the clot consists of those re- 
Fig. 2. Temporal evolution of fibrin concentration during the formation, growth, and lysis of a clot in quiescent plasma (with platelets) on a thrombogenic plane.

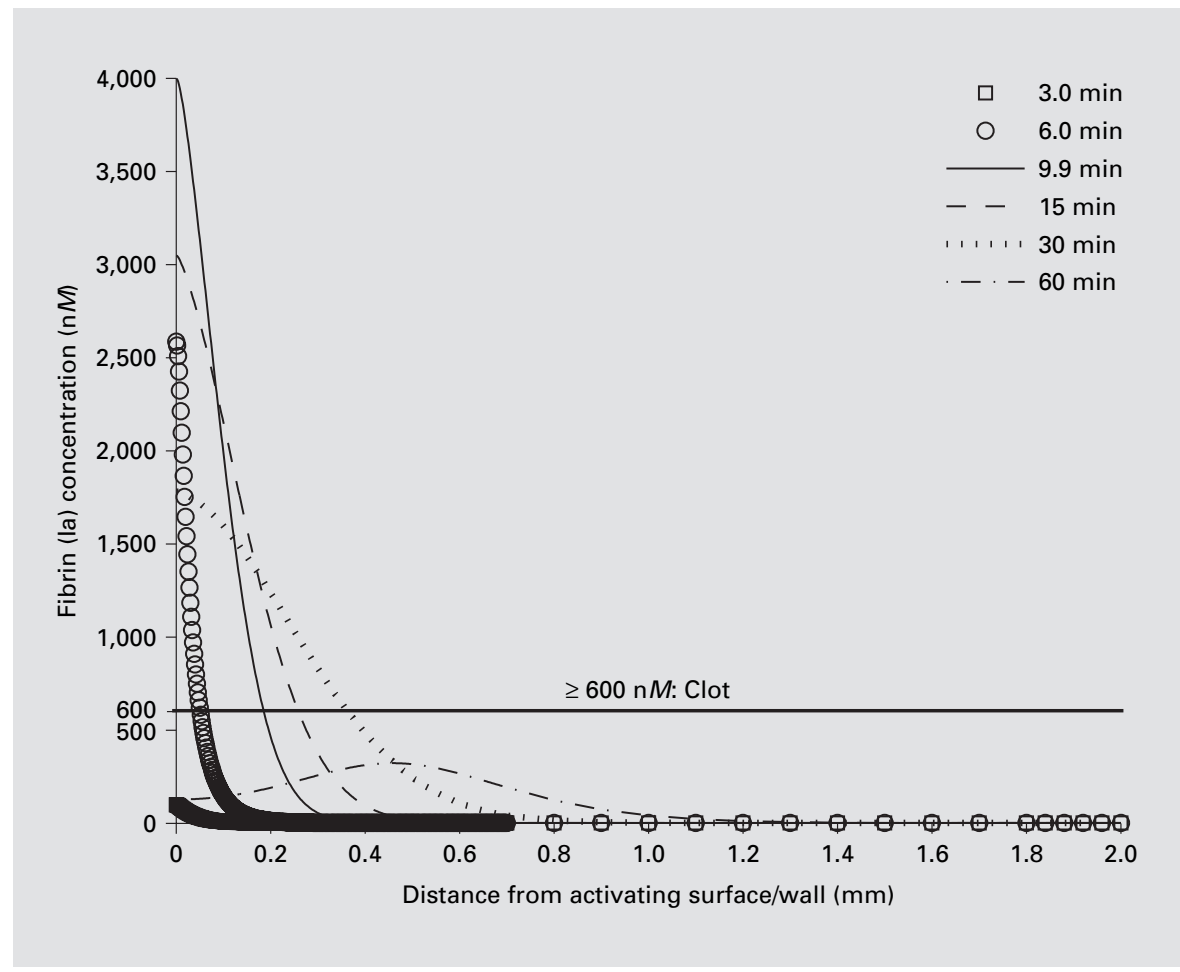

gions where $[\mathrm{Ia}] \geq 600 \mathrm{nM}$ ) are recorded in a 2-mm-thick domain (one dimensional case) over a time period of $60 \mathrm{~min}$, and the results are given in figures 1 and 2, respectively. The time for the appearance of clot is found to be $5.1 \mathrm{~min}$ from figure 1. Figure 2 shows that the clot grows to a maximum thickness of around $0.4 \mathrm{~mm}, 30 \mathrm{~min}$ after the thrombogenic stimuli are applied, before eventually dissolving, due to fibrinolysis, approximately 52 min after the application of stimuli (fig. 1). These results are in keeping with an intuitive expectation of the process of formation, growth, and lysis of a clot.

The case of time varying (oscillatory pressure gradient of amplitude $2,107.2 \mathrm{~N} / \mathrm{m}^{3}$ and frequency $2 \mathrm{~Hz}$ ) fully developed Poiseuille-type flow of blood (density of blood is $1,053.6 \mathrm{~kg} / \mathrm{m}^{3}$ ) in a cylindrical domain (of radius $\mathrm{R}=$ $0.005 \mathrm{~m}$ ) involves solving the reaction-convection-diffusion equations (and the condition for platelet activation) in conjunction with the balance laws for mass, linear and angular momentum, and energy. As a first step, however, we consider only constitutive equations for whole blood and clot (which has a viscosity that is 10 times that of whole blood), and consider a simple criterion for switching between these two models based on the extent of platelet activation and lysis of platelet aggregates. The details pertaining to the implementation are found in the litera-

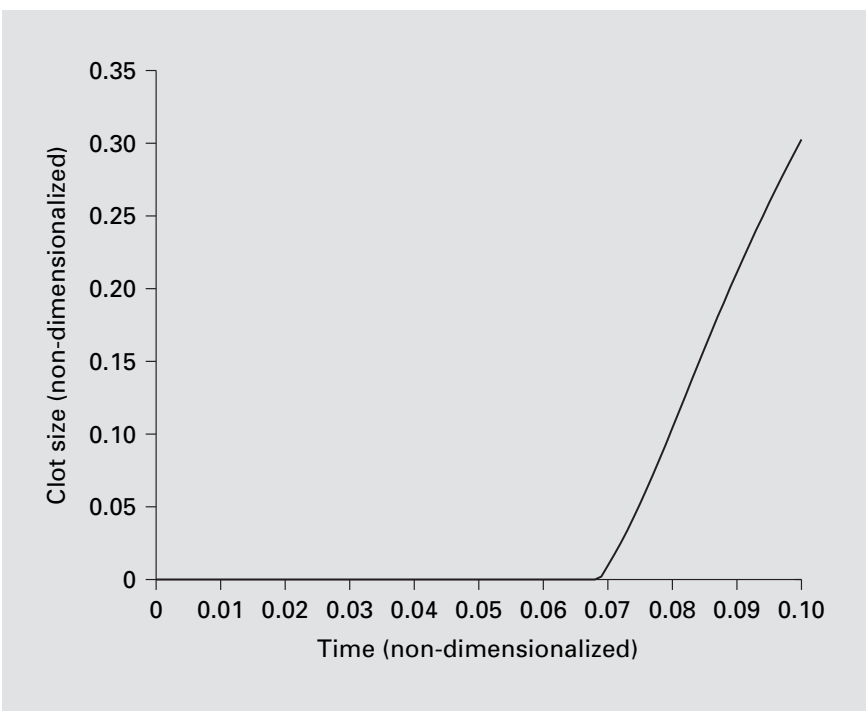

Fig. 3. Model predictions for the formation and growth of clot during the $0.05 \mathrm{~s}$ (non-dimensionalized value $=0.1$ ) after the application of the switching criterion. 


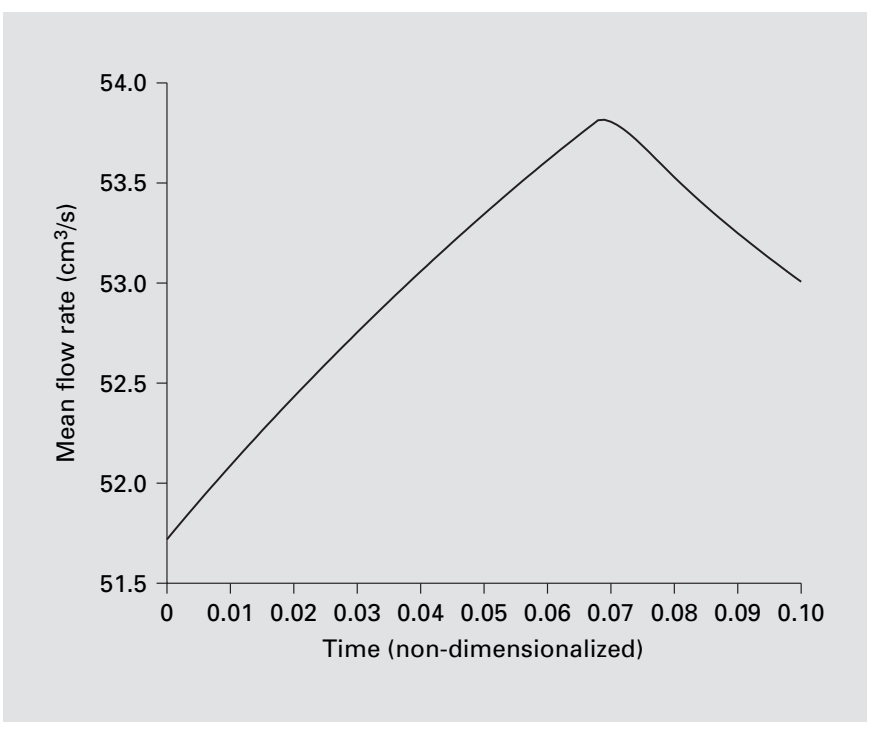

Fig. 4. Variation in mean flow rate during $0.05 \mathrm{~s}$ (non-dimensionalized value $=0.1$ ) of clot growth.

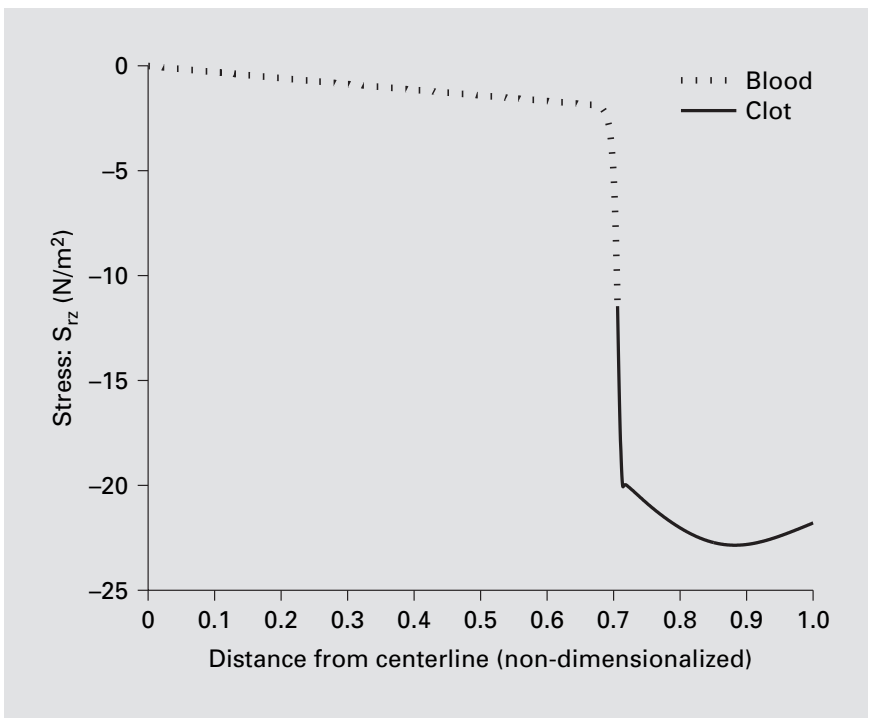

Fig. 5. Prediction of blood-clot shear stress profile $0.05 \mathrm{~s}$ after the application of the switching criterion.

ture [1], and the results (for $0.05 \mathrm{~s}$ of the simulation after the application of the switching criterion; the switching criterion is applied upon the onset of oscillatory flow) are given in figures 3-7. The model makes predictions regarding the formation and growth of the clot (the blood-clot interface is the free boundary that moves into regions occupied by blood as the clot grows; fig. 3), and the appear-

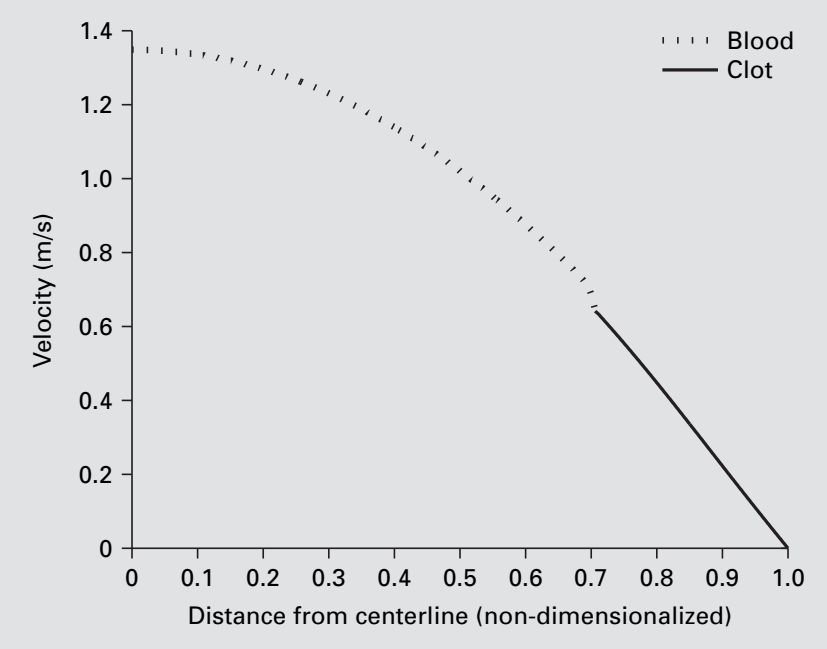

Fig. 6. Prediction of blood-clot velocity profile $0.05 \mathrm{~s}$ after the application of the switching criterion.

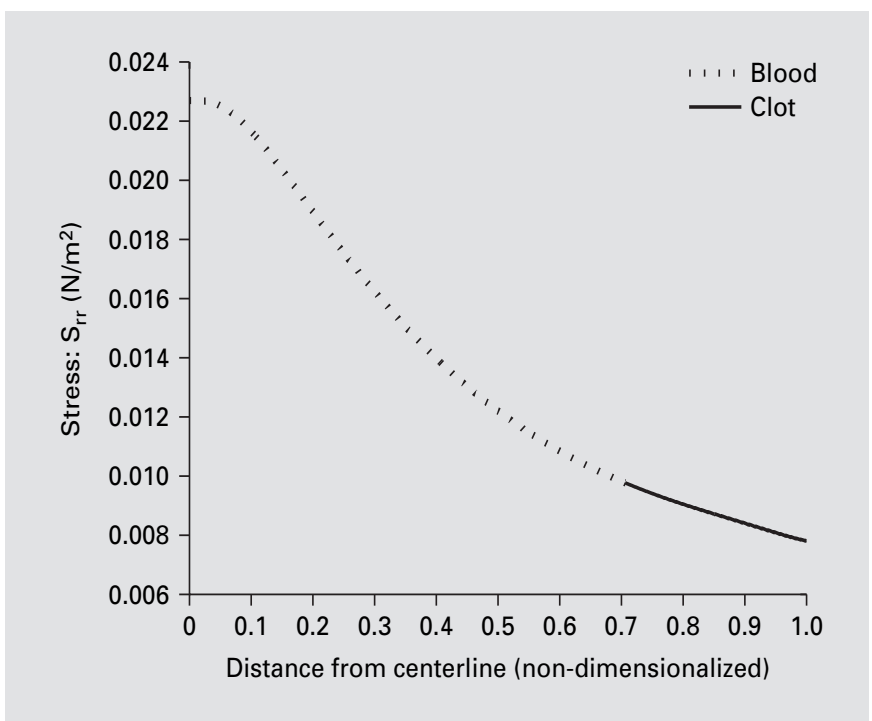

Fig. 7. Prediction of blood-clot (first) extra normal stress $\left(\mathrm{S}_{\mathrm{rr}}\right)$ profile $0.05 \mathrm{~s}$ after the application of the switching criterion.

ance of the more viscous clot leads to a reduction in the mean flow rate (fig. 4). Importantly, the model predicts that the shear stresses are much higher in the region occupied by the clot than in that occupied by blood (fig. 5), whereas neither the velocity (fig. 6) nor the extra normal stress $\left(S_{\text {rr }}\right)$ (fig. 7) show such dramatic changes across the blood-clot interface. This suggests the possibility of 
thromboembolus generation at higher pressure gradients (as the clot can rupture if shear stresses in the region it occupies become high enough [41], and the fragments can embolize distally). This tentative hypothesis must be developed and confirmed with detailed numerical studies involving the entire model.

\section{Limitations and Extensions of the Framework}

Regarding deficiencies related to the modeling, the biochemical reactions that have been taken into account are incomplete; the intrinsic coagulation pathway has not been modeled. Also, the rheological model is quite rudimentary. Blood is modeled as a single homogenized continuum, while it is in reality a complex heterogeneous mixture. Treating blood as a single constituent continuum does not allow us to track the rheology of the individual constituents. This can be redressed by developing a mixture theory [55-57] model to describe blood. The clot has also been modeled as a single homogenized continuum, while in fact it is a plasma-filled composite. Both blood and clot are modeled as rate-type shear thinning viscoelastic fluids with a very similar constitutive structure, but with different viscosities. This is an oversimplification and it might be necessary to develop very distinct constitutive models for both blood and clot. All these generalizations will be considered in subsequent studies.

\section{Acknowledgement}

M. Anand and K.R. Rajagopal thank the National Energy Technology Laboratory of the U.S. Department of Energy for its support of this work through the University Partnership Program.

\section{References}

1 Anand M, Rajagopal K, Rajagopal KR: A model incorporating some of the mechanical and biochemical factors underlying clot formation and dissolution in flowing blood. $\mathrm{J}$ Theoret Med 2003;5:183-218.

2 Virchow R: Über den Faserstoff: V. Phlogose und Thrombose im Gefäßsystem; Gesammelte Abhandlungen zur wissenschaftlichen Medicin (1st Ed). Frankfurt am Main, Verlag v. Meidinger, Sohn and Corp, 1856, p 458ff.

3 Goldsmith HL, Turitto VT: Rheological aspects of thrombosis and hemostasis: basic principles and application. Thromb Haemost 1986;55:415-435

- 4 Turitto VT, Hall CL: Mechanical factors affecting hemostasis and thrombosis. Thromb Res 1998;92:S25-S31.

-5 Lowe GDO: Rheological influences on thrombosis. Baillieres Clin Haematol 1999;12:435449 .

-6 Kuharsky AL, Fogelson AL: Surface mediated control of blood coagulation: the role of binding site densities and platelet deposition. Biophys J 2001;80:1050-1074.

-7 Sorensen EN, Burgreen GW, Wagner WR, Antaki JF: Computational simulation of platelet deposition and activation. I. Model development and properties. Ann Biomed Eng 1999; 27:436-448.

-8 Kane WH, Davie EW: Blood coagulation factors V and VIII: structural and functional similarities and their relationship to hemorrhagic and thrombotic disorders. Blood 1988;71: 539-555.
9 Nichols WC, Seligsohn U, Zivelin A, Terry VH, Hertel CE, Wheatley MA, Moussali MJ, Hauri HP, Ciavarella N, Kaufman RJ, Ginsburg D: Mutations in the ER-Golgi intermediate compartment protein ERGIC-53 cause combined deficiency of coagulation factors $\mathrm{V}$ and VIII. Cell 1998;93:61-70.

10 Hoffman R, Benz EJ, Shattil SJ, Furie B, Cohen HJ, Silberstein LE, McGlave P (eds): Hematology: Basic Principles and Practice, ed 3. New York, Churchill Livingstone, 2000.

11 Chien S, Usami S, Taylor HM, Lundberg JL, Gregersen MI: Effect of hematocrit and plasma proteins on human blood rheology at low shear rates. J Appl Physiol 1966;21:81-87.

12 Evans EA, Hochmuth RM: Membrane viscoelasticity. Biophys J 1976;16:1-11.

13 Schmid-Schönbein GW, Sung KL, Tözeren H, Skalak R, Chien S: Passive mechanical properties of human leukocytes. Biophys J 1981;36: 243-256.

14 Lasslo A (ed): Blood Platelet Function and Medicinal Chemistry. New York, Elsevier Biomedical, 1984.

15 Yamazaki H, Mustard JF (eds): Platelet Activation. Orlando, FL, Academic Press, 1987.

16 Ware AJ, Coller BS: Platelet morphology, biochemistry, and function; in Beutler E, Lichtman MA, Coller BS, Kipps TJ (eds): Williams Hematology, ed, 5. McGraw Hill Inc, 1995, pp 1161-1201.

17 Baumgartner HR: The role of blood flow in platelet adhesion, fibrin deposition and formation of mural thrombi. Microvasc Res 1973;5: 167-179.
18 Weiss HJ, Turitto VT, Baumgartner HR: Effect of shear rate on platelet interaction with subendothelium in citrated and native blood. I. Shear rate-dependent decrease of adhesion in von Willebrand's disease and the BernardSoulier syndrome. J Lab Clin Med 1978;92: 750-764.

19 Tschopp TB, Baumgartner HR, Silberbauer K, Sinzinger H: Platelet adhesion and platelet thrombus formation on subendothelium of human arteries and veins exposed to flowing blood in vitro - comparison with rabbit aorta. Haemostasis 1979;8:19-29.

20 Turitto VT, Baumgartner HR: Platelet interaction with subendothelium in flowing rabbit blood: effect of blood shear rate. Microvasc Res 1979;17:38-54.

21 Turitto VT, Weiss HJ, Baumgartner HR: The effect of shear rate on platelet interaction with subendothelium exposed to citrated human blood. Microvasc Res 1980;19:352-365.

22 Gailani D, Broze GJ: Factor XI activation in a revised model of blood coagulation. Science 1991;253:909-912.

23 Nemerson Y: Tissue factor and hemostasis. Blood 1988;71:1-8

24 Rapaport SI, Proctor RR, Patch MJ, Yettra M: The mode of inheritance of PTA deficiency: evidence for the existence of major PTA deficiency and minor PTA deficiency. Blood 1961; 18:149-165.

25 Lawson JH, Kalafatis M, Stram S, Mann KG: A model for the tissue factor pathway to thrombin. I. An empirical study. J Biol Chem 1994; 269:23357-23366. 
-26 Zarnitsina VI, Pokhilko AV, Ataullakhanov FI: A mathematical model for the spatio-temporal dynamics of intrinsic pathway of blood coagulation. I. The model description. Thromb Res 1996;84:225-236.

27 Zarnitsina VI, Pokhilko AV, Ataullakhanov FI: A mathematical model for the spatio-temporal dynamics of intrinsic pathway of blood coagulation. II. Results. Thromb Res 1996;84: 333-344.

28 Ataullakhanov FI, Krasotkina YV, Sarbash VI, Volkova RI, Sinauridse EI, Kondratov AY: Spatio-temporal dynamics of blood coagulation and pattern formation. An experimental study. Int J Bifurcation Chaos 2002;12:19691983.

-29 Ataullakhanov FI, Zarnitsina VI, Pokhilko AV, Lobanov AI, Morozova OL: Spatio-temporal dynamics of blood coagulation and pattern formation. A theoretical approach. Int $\mathbf{J}$ Bifurcation Chaos 2002;12:1985-2002.

-30 Panteleev MA, Zarnitsina VI, Ataullakhanov FI: Tissue factor pathway inhibitor. A possible mechanism of action. Eur J Biochem 2002; 269:2016-2031.

- 31 Ataullakhanov FI, Molchanova DA, Pokhilko $\mathrm{AV}$ : Mathematical model of the blood coagulation system: intrinsic pathway. Biofizika 1995; 40:434-442.

32 Kondratovich AY, Pokhilko AV, Ataullakhanov FI: Spatiotemporal dynamics of contact activation factors of blood coagulation. Biochim Biophys Acta (General Subjects) 2002; 1569:86-104.

- 33 Ataullakhanov FI, Guria GT, Sarbash VI, Volkova RI: Spatio-temporal dynamics of clotting and pattern formation in human blood. Biochim Biophys Acta (General Subjects) 1998;1425:453-468.

34 Blömback B: Fibrinogen and fibrin - proteins with complex roles in hemostasis and thrombosis. Thromb Res 1996;83:1-75.
35 Bauer KA, Rosenberg RG: Control of coagulation reactions; in Beutler E, Lichtman K, Coller BS, Kipps TJ (eds): Williams Hematology, ed 5. McGraw Hill Inc, 1995, pp 1239-1251.

36 Jesty J, Nemerson Y: The pathways of blood coagulation; in Beutler E, Lichtman MA, Coller BS, Kipps TJ (eds): Williams Hematology (5th Ed). McGraw Hill Inc, 1995, pp 1222 1238.

37 Lawson JH, Butenas S, Ribarik H, Mann KG: Complex-dependent inhibition of Factor VIIa by antithrombin III and heparin. J Biol Chem 1993;268:767-770.

38 Tippe A, Müller-Mohnssen EN: Shear dependence of the fibrin coagulation kinetics in-vitro. Thromb Res 1993;72:379-388.

39 Reininger AJ, Heinzmann U, Reininger CB Friedrich P, Wurzinger LJ: Flow mediated fibrin thrombus formation in an endotheliumlined model of arterial branching. Thromb Res 1994;74:629-641.

40 Francis CW, Marder VJ: Mechanisms of fibrinolysis; in Beutler E, Lichtman MA, Coller BS, Kipps TJ (eds): Williams Hematology, ed 5. McGraw Hill Inc, 1995, pp 1252-1260.

41 Riha P, Wang X, Liao R, Stoltz JF: Elasticity and fracture strain of whole blood clots. Clin Hemorheol Microcirc 1999;21:45-49.

42 Charm S, Kurland G: Viscometry of human blood for shear rates of $0-100,000 \mathrm{~s}^{-1}$. Nature 1965;206:617-618.

43 Thurston GB: Viscoelasticity of human blood. Biophys J 1972;12:1205-1217.

44 Chien S, Sung KLP, Skalak R, Usami S, Tözeren AL: Theoretical and experimental studies on viscoelastic properties of erythrocyte membrane. Biophys J 1978;24:463-487.

45 Thurston GB: Frequency and shear rate dependence of viscoelasticity of blood. Biorheology 1973;10:375-381.

46 Yeleswarapu KK: Evaluation of Continuum Models for Characterizing the Constitutive Behavior of Blood; PhD dissertation, University of Pittsburgh Pittsburgh, PA, 1996.
47 Rajagopal KR, Srinivasa AR: A thermodynamic framework for rate type fluid models. J Non-Newtonian Fluid Mechanics 2000;88: 207-227.

48 Anand M, Rajagopal KR: A shear-thinning viscoelastic fluid model for describing the flow of blood. Int J Cardiovasc Med Science 2004;4: 59-68.

49 Oldroyd JG: On the formulation of rheological equations of state. Proc R Soc Lond A Math Phys Sci 1950;200:523-541.

50 Brown CH, Lemuth RF, Hellums JD, Leverett LB, Alfrey CP: Response of human platelets to shear stress. Trans ASAIO 1975;21:35-39.

51 Brown CH, Leverett LB, Lewis CW, Alfrey CP, Hellums JD: Morphological, biochemical and functional changes in human platelets subjected to shear stresses. J Lab Clin Med 1975;86: 462-471.

52 Anand M, Rajagopal KR: A mathematical model to describe the change in the constitutive character of blood due to platelet activation. C R Mecanique 2002;330:557-562.

53 Wurzinger LJ, Opitz R, Wolf M, SchmidSchönbein H: Shear induced platelet activation - a critical reappraisal. Biorheology 1985; 22:399-413.

54 Anand M, Rajagopal K, Rajagopal KR: A viscoelastic liquid model for describing the flow of a coarse ligated plasma clot. Theoretical and Computational Fluid Dynamics 2005.

55 Truesdell C: Sulle basi della Termodinamica Rendiconti Academia Nazionale dei Lincei 1957:22:33-38.

56 Truesdell C: Sulle basi della Termodinamica. Rendiconti Academia Nazionale dei Lincei 1957;22:158-166.

57 Rajagopal KR, Tao L: Mechanics of mixtures. Singapore, World Scientific Publishing Co, 1995. 